\title{
On Asymptotic Stability of Linear Control Systems
}

\section{Şerife Yılmaz*, Taner Büyükköroğlu, Vakif Dzhafarov}

Department of Mathematics, Faculty of Science, Anadolu University, Eskisehir, Turkey

Email: serifeyilmaz@anadolu.edu.tr, tbuyukkoroglu@anadolu.edu.tr, vcaferov@anadolu.edu.tr

Received 10 November 2014; revised 6 December 2014; accepted 25 December 2014

Copyright (C) 2015 by authors and Scientific Research Publishing Inc.

This work is licensed under the Creative Commons Attribution International License (CC BY).

http://creativecommons.org/licenses/by/4.0/

c) (i) Open Access

\section{Abstract}

Asymptotic stability of linear systems is closely related to Hurwitz stability of the system matrices. For uncertain linear systems we consider stability problem through common quadratic Lyapunov functions (CQLF) and problem of stabilization by linear feedback.

\section{Keywords}

Common Quadratic Lyapunov Functions, Uncertain System, Gradient Method, Bendixson Theorem

\section{Introduction}

Let linear uncertain system

$$
\dot{x}=A x, \quad A \in \operatorname{conv}\left\{A_{1}, A_{2}, \cdots, A_{N}\right\}
$$

be given where $x=x(t) \in \mathbb{R}^{n}, A_{i} \quad(i=1,2, \cdots, N)$ are $n \times n$ real matrices. Consider the following matrix inequalities

$$
A_{i}^{\mathrm{T}} P+P A_{i}<0 \quad(i=1,2, \cdots, N)
$$

where $P>0$ and the symbol " $>$ " stands for positive definiteness. The matrix $P$ is called a common solution to (2).

If the system (2) has a common $P>0$ solution, then this system is uniformly asymptotically stable [1].

The problem of existence of common positive definite solution $P$ of (2) has been studied in a lot of works (see [1]-[7] and references therein). Numerical solution for common $P$ via nondifferentiable convex optimization has been discussed in [8].

*Corresponding author. 
In the first part of the paper we treat the problem (2) as a nonconvex optimization problem (minimization of a convex function under nonconvex constraints) and apply a modified gradient method. The comparison with [8] shows that our approach gives better result in some cases.

In the second part we consider the stabilization problem, i.e. the following question: for the affine family

$$
\{A(q): q \in R\}
$$

where $R \subset \mathbb{R}^{l}$ is a box, is there a stable member? We consider a sufficient condition which follows from the Bendixson theorem [9].

\section{Gradient Method}

According to [2], let $\mathcal{S}$ be the set (subspace) of $(n \cdot N) \times(n \cdot N)$ dimensional symmetric block-diagonal matrices of the form $R \oplus R \oplus \cdots \oplus R$ where $R$ is symmetric.

Let $Z_{1}, Z_{2}, \cdots, Z_{r}$ be a basis of $\mathcal{S}, r=n(n+1) / 2$,

$$
\begin{aligned}
& Q_{i}=\left(-Z_{i}\right) \oplus\left(A_{1}^{\mathrm{T}} Z_{i}+Z_{i} A_{1}\right) \oplus \cdots \oplus\left(A_{N}^{\mathrm{T}} Z_{i}+Z_{i} A_{N}\right) \\
& \phi(x)=\phi\left(x_{1}, x_{2}, \cdots, x_{r}\right)=\lambda_{\text {max }}\left(\sum_{i=1}^{r} x_{i} Q_{i}\right)
\end{aligned}
$$

Then $\left\{A_{1}, A_{2}, \cdots, A_{N}\right\}$ has CQLF $\Leftrightarrow$ there exists $x_{*} \in \mathbb{R}^{r}$ such that $\phi\left(x_{*}\right)<0$. In this case the matrix $P\left(x_{*}\right)$ is a common solution to (2) where

$$
P(x)=\left(\begin{array}{cccc}
x_{1} & x_{2} & \cdots & x_{n} \\
x_{2} & x_{n+1} & \cdots & x_{2 n-1} \\
\vdots & \vdots & \ddots & \vdots \\
x_{n} & x_{2 n-1} & \cdots & x_{r}
\end{array}\right) .
$$

The function $\phi(x)$ is positive homogenous $(\phi(\alpha x)=\alpha \phi(x)$ for all $\alpha \geq 0)$. Therefore the vector $x$ can be restricted to the condition $\|x\|=1$. The advantage of the restriction $\|x\|=1$ shows the following proposition.

Proposition 1. Let $S=\left\{x \in \mathbb{R}^{r}: x=1\right\}$ be the unit sphere, let the function $f: \mathbb{R}^{r} \rightarrow \mathbb{R}$ be positive homogeneous $(f(\lambda x)=\lambda f(x)$ for all $\lambda>0)$ and be differentiable at $a \in S$. Assume that $f(a)>0$. Then $\langle g, a\rangle<0$ where $g=-\left.\nabla f(x)\right|_{x=a}, \nabla$ denotes the gradient and $\langle\cdot, \cdot\rangle$ denotes the scalar product.

Proof: Since $f$ is positive homogeneous, it increases in the direction of the vector $a$ : for $\lambda>1$,

$$
f(\lambda a)=\lambda f(a)>f(a) .
$$

Therefore the directional derivative of $f$ at $a$ in the direction of $a$ is positive $D_{a} f(a)>0$.

On the other hand

$$
D_{a} f(a)=\langle\nabla f, a\rangle
$$

and

$$
\langle\nabla f, a\rangle>0 \text { or }\langle-\nabla f, a\rangle<0 \text { or }\langle g, a\rangle<0 .
$$

Proposition 1 shows that under its assumption the minus gradient vector at the point $a$ is directed into the unit ball (Figure 1).

Consider the following optimization problem

$$
\begin{gathered}
\phi(x) \rightarrow \text { minimize } \\
\text { subject to } x=1 .
\end{gathered}
$$




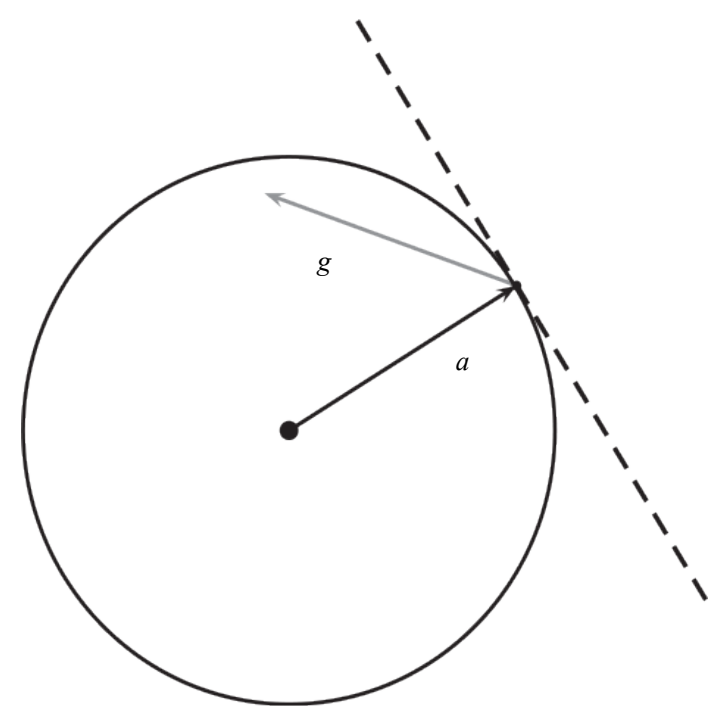

Figure 1. The direction $(g)$ of the minus gradient.

Since the matrix $\sum_{i=1}^{r} x_{i} Q_{i}$ is symmetric, the function $\phi(x)$ (3) can be written as

$$
\phi(x)=\max _{\|u\|=1} u^{\mathrm{T}}\left(\sum_{i=1}^{r} x_{i} Q_{i}\right) u .
$$

The gradient vector of $\phi(x)$ at a point $a$ is:

$$
\left.\nabla \phi(x)\right|_{x=a}=\left(u^{\mathrm{T}} Q_{1} u, u^{\mathrm{T}} Q_{2} u, \cdots, u^{\mathrm{T}} Q_{r} u\right)
$$

where $u$ is the unit eigenvector of $\sum_{i=1}^{r} a_{i} Q_{i}$ corresponding to the simple maximum eigenvalue [2].

Well-known gradient algorithm in combination with Proposition 1 gives the following.

\section{Algorithm 1.}

Step 1. Take an initial point $x^{0}=S$. Compute $\phi\left(x^{0}\right)$. If $\phi\left(x^{0}\right) \geq 0$, find $t$ such that the line

$$
l(t)=x^{0}-\left.t \cdot \nabla \phi(x)\right|_{x=x^{0}}
$$

intersects the unit sphere $S$ (Figure 2).

Step 2. Take $x^{1}=x^{0}-\left.t_{*} \cdot \nabla \phi(x)\right|_{x=x^{0}}$ where $t_{*}$ satisfies the condition $\left\|l\left(t_{*}\right)\right\|=1$. If $\phi\left(x^{1}\right)<0, x^{1}$ is required point. Otherwise find $t$ such that the line $l(t)=x^{1}-\left.t \cdot \nabla \phi(x)\right|_{x=x^{1}}$ intersects the unit sphere and repeat the procedure.

Example 1. Consider the switched system

$$
\dot{x} \in\left\{A_{1}, A_{2}\right\} x
$$

where

$$
A_{1}=\left(\begin{array}{ccc}
-4 & -1 & 3 \\
-3 & -2 & 2 \\
3 & 0 & -3
\end{array}\right), \quad A_{2}=\left(\begin{array}{ccc}
-8 & -3 & 1 \\
9 & 2 & 0 \\
6 & 3 & -6
\end{array}\right)
$$

are Hurwitz stable matrices. Let

$$
Z_{1}=\left(\begin{array}{lll}
1 & 0 & 0 \\
0 & 0 & 0 \\
0 & 0 & 0
\end{array}\right), \quad Z_{2}=\left(\begin{array}{lll}
0 & 1 & 0 \\
1 & 0 & 0 \\
0 & 0 & 0
\end{array}\right), \quad Z_{3}=\left(\begin{array}{lll}
0 & 0 & 1 \\
0 & 0 & 0 \\
1 & 0 & 0
\end{array}\right),
$$




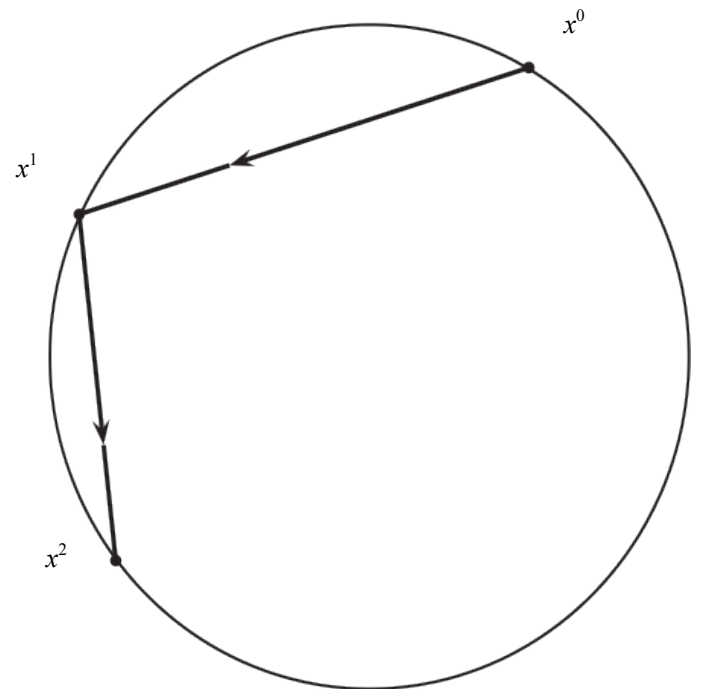

Figure 2. Searching on the unit sphere.

$$
Z_{4}=\left(\begin{array}{lll}
0 & 0 & 0 \\
0 & 1 & 0 \\
0 & 0 & 0
\end{array}\right), Z_{5}=\left(\begin{array}{lll}
0 & 0 & 0 \\
0 & 0 & 1 \\
0 & 1 & 0
\end{array}\right) \text { and } Z_{6}=\left(\begin{array}{lll}
0 & 0 & 0 \\
0 & 0 & 0 \\
0 & 0 & 1
\end{array}\right)
$$

For $i=1,2, \cdots, 6$

$$
Q_{i}=\left(-Z_{i}\right) \oplus\left(A_{1}^{\mathrm{T}} Z_{i}+Z_{i} A_{1}\right) \oplus\left(A_{2}^{\mathrm{T}} Z_{i}+Z_{i} A_{2}\right) .
$$

Take the initial point $x^{0}=(1 / \sqrt{3}, 0,0,1 / \sqrt{3}, 0,1 / \sqrt{3})^{\mathrm{T}}$, then

$$
P\left(x^{0}\right)=\left(\begin{array}{ccc}
1 / \sqrt{3} & 0 & 0 \\
0 & 1 / \sqrt{3} & 0 \\
0 & 0 & 1 / \sqrt{3}
\end{array}\right)
$$

is positive definite. Eigenvalues of the matrix

$$
\sum_{i=1}^{6} x_{i}^{0} Q_{i}=\frac{1}{\sqrt{3}} \cdot Q_{1}+0 \cdot Q_{2}+0 \cdot Q_{3}+\frac{1}{\sqrt{3}} \cdot Q_{4}+0 \cdot Q_{5}+\frac{1}{\sqrt{3}} \cdot Q_{6}
$$

are $-12.507,-5.364,4.015,-0.224,-0.577,-8.566,-1.601$.

Maximum eigenvalue 4.015 is simple and the corresponding unit eigenvector is

$$
v=(0,0,0,0,0,0,-0.317,-0.911,-0.261)^{\mathrm{T}} .
$$

Gradient of the function $\phi$ at $x^{0}$ is

$$
\left.\nabla \phi(x)\right|_{x=x^{0}}=(3.189,6.162,0.671,-8.537,-8.049,-1.607)^{\mathrm{T}} \text {. }
$$

The vector $x^{1}=x^{0}-\left.t \cdot \nabla \phi(x)\right|_{x=x^{0}}$ should be on the six dimensional unit sphere. Therefore $t=0.0425$ and

$$
x^{1}=(0.7129,0.2620,0.0285,0.2143,-0.3422,0.5090)^{\mathrm{T}} .
$$

After 9 steps, we get $\phi\left(x^{9}\right)<0$ where

$$
x^{9}=(0.7950,0.2183,-0.0623,0.2185,-0.1254,0.5028)^{\mathrm{T}},
$$




$$
P\left(x^{9}\right)=\left(\begin{array}{ccc}
0.7950 & 0.2183 & -0.0623 \\
0.2183 & 0.2185 & -0.1254 \\
-0.0623 & -0.1254 & 0.5028
\end{array}\right) .
$$

$P\left(x^{9}\right)$ is a common positive definite solution for $A_{1}^{\mathrm{T}} P\left(x^{9}\right)+P\left(x^{9}\right) A_{1}<0$ and $A_{2}^{\mathrm{T}} P\left(x^{9}\right)+P\left(x^{9}\right) A_{2}<0$.

The same problem solved by the algorithm from [8] gives answer only after 70 steps. We have solved a number of examples using the above gradient algorithm and by the algorithm from [8]. These examples show that this algorithm is faster than the algorithm from [8] in some cases.

As the comparison with the algorithm from [8] is concerned, the algorithm from [8] at each step uses the gradient only one maximum eigenvalue function, i.e. at 1 step it uses the gradient of $P \rightarrow \lambda_{\max }\left(A_{1}^{\mathrm{T}} P+P A_{1}\right)$, at 2 step the gradient of $P \rightarrow \lambda_{\max }\left(A_{2}^{\mathrm{T}} P+P A_{2}\right)$ and so on. This procedure delays the convergence. In our algorithm we use the function $P \rightarrow \max _{i}\left(\lambda_{\max }\left(A_{i}^{\mathrm{T}} P+P A_{i}\right)\right)$ and the corresponding gradient direction decreases the greates maximum eigenvalue.

On the other hand an obviously advantage of the method from [8] is the choose of the step size, which is given by an exact formula, whereas our step size is determined by the intersection of the corresponding rays with the unit sphere.

\section{Sufficient Condition for a Stable Member}

In this section we consider a sufficient condition for a stable member which is obtained by using Bendixson's theorem.

If a matrix is symmetric then it is stable if and only if it is negative definite. Therefore if a family consists of symmetric matrices then searching for stable element is equivalent to the searching for negative definite one.

On the other hand every real $n \times n$ matrix $A$ can be decomposed

$$
\begin{aligned}
& A=B+C, \\
& B=\frac{1}{2}\left(A+A^{\mathrm{T}}\right), \\
& C=\frac{1}{2}\left(A+A^{\mathrm{T}}\right) .
\end{aligned}
$$

where $B$ is symmetric and $C$ is skew-symmetric. Bendixson's theorem gives important inequalities for the eigenvalues of $A, B$ and $C$.

Theorem 1. ([9], p. 40) If $A$ is an $n \times n$ matrix, $B=\frac{1}{2}\left(A+A^{\mathrm{T}}\right)$ and $\lambda_{1}, \lambda_{2}, \cdots, \lambda_{n} \quad\left(\left|\lambda_{1}\right| \geq\left|\lambda_{2}\right| \geq \cdots \geq\left|\lambda_{n}\right|\right)$, $\mu_{1} \geq \mu_{2} \geq \cdots \geq \mu_{n}$ are the eigenvalues of $A, B$ then

$$
\mu_{n} \leq \operatorname{Re}\left(\lambda_{i}\right) \leq \mu_{1} \quad(i=1,2, \cdots, n)
$$

Bendixson's theorem leads to the following.

Proposition 2. Let the family $\{A(q): q \in R\}$ be given and $B(q)$ is the symmetric part of $A(q)$. Then

1) If there exists $q_{*} \in R$ such that $B\left(q_{*}\right)$ is Hurwitz stable then $A\left(q_{*}\right)$ is also Hurwitz stable,

2) If there exists $q_{*} \in R$ such that $B\left(q_{*}\right)$ is positive stable (all eigenvalues lie in the open right half plane) then $A\left(q_{*}\right)$ is also positive stable.

Proposition 2 gives a sufficient condition for the existence of a stable element.

In the case of affine family

$$
A(q)=A_{0}+q_{1} A_{1}+q_{2} A_{2}+\cdots+q_{l} A_{l}
$$

where $q=\left(q_{1}, q_{2}, \cdots, q_{l}\right)^{\mathrm{T}} \in R, R$ is a box or $R=\mathbb{R}^{l}$, the searching procedure for stable element in $B(q)$ can be effectively solved by powerful tools of Linear Matrix Inequalities (Matlab's LMI Toolbox).

In the non-affine case of the family $A(q)$ the gradient algorithm for a stable element in $B(q)$ is applicable.

Example 2. Consider affine family 


$$
A(q)=\left(\begin{array}{ccc}
6-3 q_{1}-q_{2}-q_{3} & 2+q_{1}-4 q_{3} & -2-5 q_{1}-q_{2}-q_{3} \\
5+q_{1}+3 q_{2}-q_{3} & 8-2 q_{1}-2 q_{2}+2 q_{3} & 3+q_{1}-3 q_{3} \\
5+5 q_{1}-q_{2}+2 q_{3} & -4 q_{1}-5 q_{2}+q_{3} & -2 q_{1}-q_{2}
\end{array}\right)
$$

$q_{i} \in[-10,10] \quad(i=1,2,3)$. Then

$$
B(q)=\left(\begin{array}{ccc}
6-3 q_{1}-q_{2}-q_{3} & \left(7+2 q_{1}+3 q_{2}-5 q_{3}\right) / 2 & \left(3-2 q_{2}+q_{3}\right) / 2 \\
\left(7+2 q_{1}+3 q_{2}-5 q_{3}\right) / 2 & 8-2 q_{1}-2 q_{2}+2 q_{3} & \left(3-3 q_{1}-5 q_{2}-2 q_{3}\right) / 2 \\
\left(3-2 q_{2}+q_{3}\right) / 2 & \left(3-3 q_{1}-5 q_{2}-2 q_{3}\right) / 2 & -2 q_{1}-q_{2}
\end{array}\right) .
$$

LMI method applied to the matrix inequality problem $B(q)<0$ gives the value within a few seconds

$$
q_{*}=(9.4591,-3.5180,-0.0354)^{\mathrm{T}}
$$

and $B\left(q_{*}\right)$, and consequently $A\left(q_{*}\right)$ is stable.

LMI method applied to the inequality $B(q)>0$ gives also

$$
\tilde{q}=(-2.6549,1.3609,0.9393)^{\mathrm{T}}
$$

so the family $A(q)$ contains positive stable matrix $A(\tilde{q})$.

We have investigated Example 2 by the algorithm from [10] and positive answer is obtained after about 100 seconds.

Example 3. Consider non-affine family

$$
A(q)=\left(\begin{array}{ccc}
q_{1} q_{2}+q_{2}-2 & -q_{1} q_{3}+q_{1}-q_{3}-9 & -3 q_{2} q_{3}+3 q_{2}+3 q_{3}-10 \\
-17-q_{1}+q_{3} & -q_{1}-4 & q_{2}-4 \\
q_{1}+5 & q_{1}+11 & q_{2}-6
\end{array}\right)
$$

$q_{i} \in[-10,10] \quad(i=1,2,3)$. Here

$$
B(q)=\left(\begin{array}{ccc}
q_{1} q_{2}+q_{2}-2 & -\frac{q_{1} q_{3}}{2}-13 & \frac{q_{1}-3 q_{2}\left(q_{3}-1\right)+3 q_{3}-5}{2} \\
-\frac{q_{1} q_{3}}{2}-13 & -q_{1}-4 & \frac{q_{1}+q_{2}+7}{2} \\
\frac{q_{1}-3 q_{2}\left(q_{3}-1\right)+3 q_{3}-5}{2} & \frac{q_{1}+q_{2}+7}{2} & q_{2}-6
\end{array}\right) .
$$

Consider the function

$$
G(q)=\lambda_{\max }(B(q))=\max _{\|v\|=1} v^{\mathrm{T}} B(q) v .
$$

We are looking for $q$ satisfying $G(q)<0$. If for some $q$ the maximal eigenvalue $\lambda_{\max }(B(q))$ is simple then $G(q)$ is differentiable at $q$ and its gradient can be easily calculated (by the analogy with (4)).

For this example, gradient method gives solution after 7 steps:

$$
q^{0}=(0,0,0)^{\mathrm{T}}, \cdots, q^{7}=(5.270,-6.252,0.959)^{\mathrm{T}}
$$

(see Table 1). The step size $t$ is chosen from the decreasing condition of the function $G(q): t$ must be chosen such that

$$
G\left(q^{k+1}\right)=G\left(q^{k}-\left.t \nabla G\right|_{q^{k}}\right)<G\left(q^{k}\right) .
$$

This example has been solved by the algorithm from [10] as well. Positive answer has been obtained only after 
Table 1. Gradient algorithm for example 3.

\begin{tabular}{ccccc}
\hline$k$ & $q^{k}$ & $\lambda_{\max }$ & multiplicity & $\left.\nabla G\right|_{q^{k}}$ \\
\hline 0 & $(0,0,0)$ & 11.079 & 1 & $(-0.452,0.208,-0.508)$ \\
1 & $(0.411,-0.189,0.462)$ & 10.632 & 1 & $(-0.332,0.655,-0.355)$ \\
2 & $(0.714,-0.785,0.786)$ & 9.910 & 1 & $(-0.482,0.930,-0.383)$ \\
3 & $(1.153,-1.632,1.135)$ & 8.634 & 1 & $(-0.719,1.173,-0.184)$ \\
4 & $(1.808,-2.700,1.303)$ & 6.712 & 1 & $(-1.061,1.303,0.291)$ \\
5 & $(2.774,-3.886,1.038)$ & 3.840 & 1 & $(-1.391,1.360,0.060)$ \\
6 & $(4.040,-5.123,0.983)$ & 0.444 & 1 & $(-1.352,1.240,0.267)$ \\
7 & $(5.270,-6.252,0.959)$ & -2.404 & & \\
\hline
\end{tabular}

55 steps. We start with $q^{0}=(0,0,0)$ and the algorithm from [10] gives another stabilizing point

$$
q^{55}=(3.2721,-2.3853,2.3818)^{\mathrm{T}} .
$$

The eigenvalues of $A\left(q^{55}\right)$ are $\lambda_{1}=-27.8402, \lambda_{2,3}=-0.004 \pm j 0.2326$.

\section{Conclusion}

In the first part of the paper, we consider the stability problem of a matrix polytope through common quadratic Lyapunov functions. We suggest a modified gradient algorithm. In the second part by using Bendixson's theorem a sufficient condition for a stable member is given.

\section{References}

[1] Liberzon, D. (2003) Switching in System and Control. Birkhäuser, Boston. http://dx.doi.org/10.1007/978-1-4612-0017-8

[2] Boyd, S. and Yang, Q. (1989) Structured and Simultaneous Lyapunov Functions for System Stability Problems. International Journal of Control, 49, 2215-2240. http://dx.doi.org/10.1080/00207178908559769

[3] Büyükköroğlu, T., Esen, Ö. and Dzhafarov, V. (2011) Common Lyapunov Functions for Some Special Classes of Stable Systems. IEEE Transactions on Automatic Control, 56, 1963-1967. http://dx.doi.org/10.1109/TAC.2011.2137510

[4] Cheng, D., Guo, L. and Huang, J. (2003) On Quadratic Lyapunov Functions. IEEE Transactions on Automatic Control, 48, 885-890. http://dx.doi.org/10.1109/TAC.2003.811274

[5] Mason, O. and Shorten, R. (2006) On the Simultaneous Diagonal Stability of a Pair of Positive Linear Systems. Linear Algebra and Its Applications, 413, 13-23. http://dx.doi.org/10.1016/j.laa.2005.07.019

[6] Narendra, K.S. and Balakrishnan, J. (1994) A Common Lyapunov Function for Stable LTI Systems with Commuting A-Matrices. IEEE Transactions on Automatic Control, 39, 2469-2471. http://dx.doi.org/10.1109/9.362846

[7] Shorten, R.N. and Narendra, K.S. (2002) Necessary and Sufficient Conditions for the Existence of a Common Quadratic Lyapunov Function for a Finite Number of Stable Second Order Linear Time-Invariant Systems. International Journal of Adaptive Control and Signal Processing, 16, 709-728. http://dx.doi.org/10.1002/acs.719

[8] Liberzon, D. and Tempo, R. (2004) Common Lyapunov Functions and Gradient Algorithms. IEEE Transactions on Automatic Control, 49, 990-994. http://dx.doi.org/10.1109/TAC.2004.829632

[9] Marcus, M. and Minc, H.A. (1992) Survey of Matrix Theory and Matrix Inequalities. Dover, New York.

[10] Polyak, B.T. and Shcherbakov, P.S. (1999) Numerical Search of Stable or Unstable Element in Matrix or Polynomial Families: A Unified Approach to Robustness Analysis and Stabilization. Robustness in Identification and Control Lecture Notes in Control and Information Sciences, 254, 344-358. http://dx.doi.org/10.1007/BFb0109879 
Scientific Research Publishing (SCIRP) is one of the largest Open Access journal publishers. It is currently publishing more than 200 open access, online, peer-reviewed journals covering a wide range of academic disciplines. SCIRP serves the worldwide academic communities and contributes to the progress and application of science with its publication.

Other selected journals from SCIRP are listed as below. Submit your manuscript to us via either submit@scirp.org or Online Submission Portal.
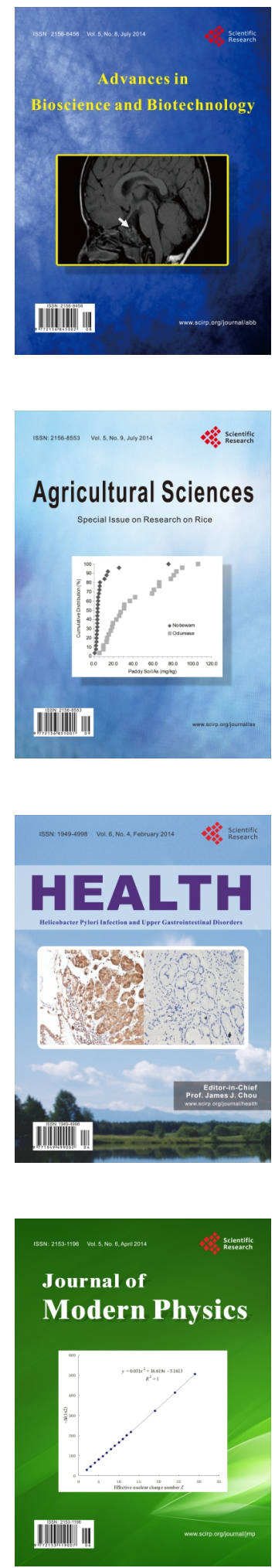
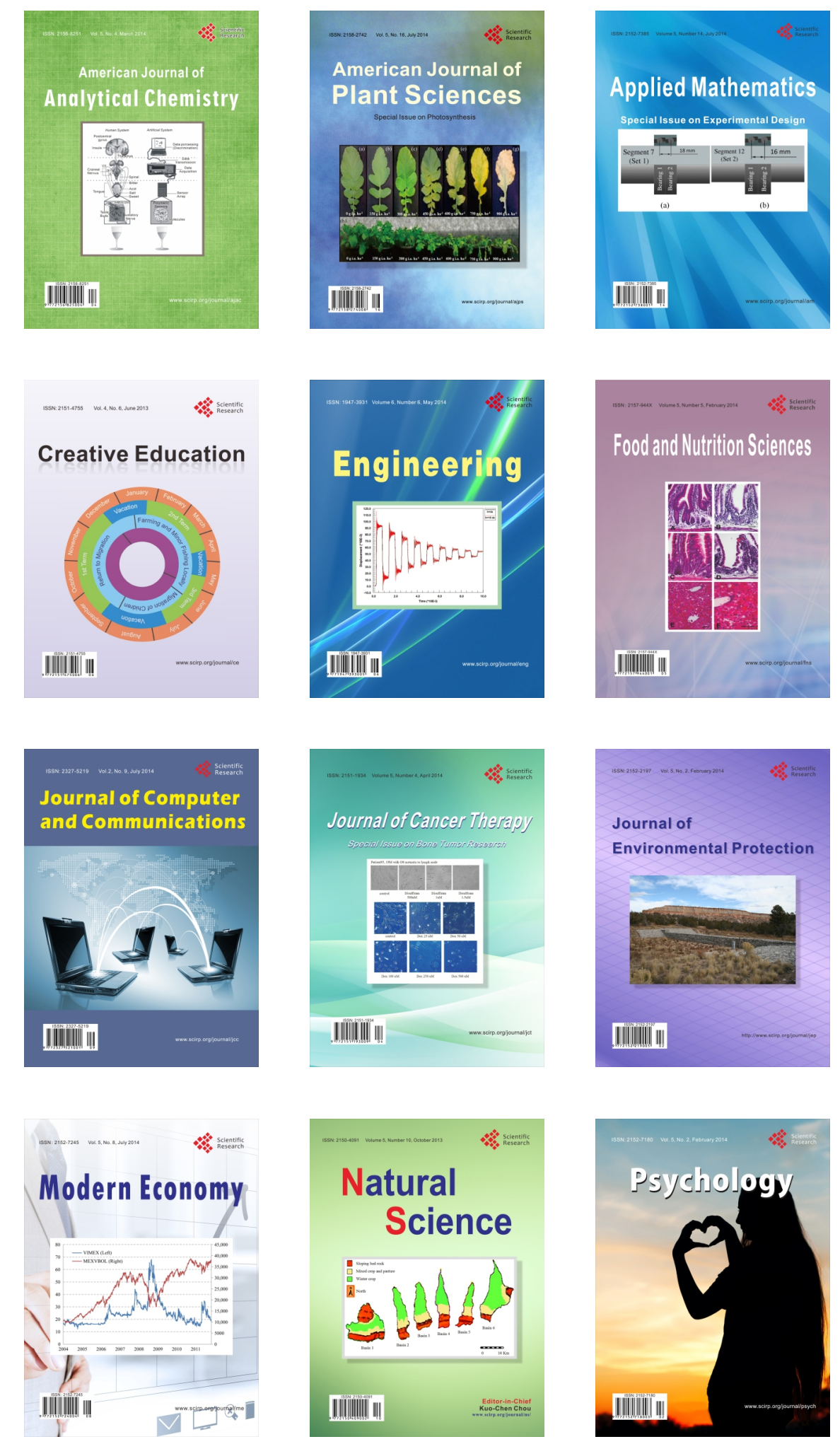